\title{
A Tentative Research of Internet of Things (IOT) in Automotive Transportation
}

\author{
Qingqiang Huang ${ }^{a}$, Guanglong Zhang, Chunxiao She \\ Guilin University of Electronic Technology, Beihai Campus, 536000, P.R. China \\ a45306174@qq.com
}

\begin{abstract}
Characterized by big data, artificial intelligence, IOT, automation, the industrial sector's transition to Industry 4.0 is much more than just a question of technology. It will affect not only individual corporate strategy but also national economic and employment policy. The Internet of Things provides a groundbreaking and reliable platform for car manufacturers by introducing entirely new layers to the traditional concept of a car. By offering a very large variety of infotainment services and connected car applications for drivers, the automotive sector has the potentiality to become a prominent IOT champion and pump up adoption of IOT cloud services.
\end{abstract}

Keywords: Application, IOT, Cloud Service, Automotive Transportation.

\section{Introduction to IOT and Automotive Cloud Services}

\subsection{Internet of Things (IOT)}

An IOT platform is a multi-layer technology that enables various straightforward provisioning, management, and automation of connected devices within the Internet of Things universe. It basically connects our hardware, however diverse, to the cloud by using flexible connectivity options, enterprise-grade security mechanisms, and broad data processing powers. For developers, an IOT platform provides a set of ready-to-use features that greatly speed up development of applications for connected devices as well as take care of scalability and cross-device compatibility.

\subsection{IOT Automotive Cloud Services}

Regarding connected car engineering, scientists build up the platform that supports standard IOT protocols, as well as allows for custom implementations. Its open (Application Programming Interface) APIs ensure seamless integration with different kinds of automotive equipment — sensors, control units, electronics — and help us introduce numerous smart features in coherence with the specific car design.

\subsubsection{Connectivity}

Connectivity deals with messaging between the cloud and the devices. That is how the devices connect to the cloud to perform different operations.

The platform supports lightweight IOT protocols for device connection, such as MQTT and COAP. It is transport-agnostic and may support any open IOT protocol. The platform allows building applications that function over any type of network connection, either persistent or intermittent.

The platform defines basic rules of communication between the platform and the devices. The protocol is fully open, asynchronous, and allows for arbitrary message formats. Furthermore, we can choose between encrypted and unencrypted channels. Use the encrypted channel to secure sensitive data or the unencrypted channel for open data.

\subsubsection{Hardware Control and Management}

The platform provides a register of digital twins, which represent things, devices, and other entities. It also allows us to store device attributes, which provide more detailed information about any characteristic of the device. In addition to simple data types, attributes can contain more complex, structured objects, such as a list of connected peripherals and their properties.

To connect to the platform, a device has to present valid credentials, such as pre-shared keys, tokens, login and password combinations, certificates, etc. The platform tracks the device throughout 
its lifecycle, from the initial provisioning and connectivity events to software updates and final decommissioning.

\subsubsection{Data Collection}

Out of the box, it is easy to use protocols to collect data from connected devices. This protocol ensures reliable data delivery with response codes, which indicate the result of data processing by the platform. Once received by the platform, the device data can be dispatched to multiple processing pipelines. In case there is any error in the middle of processing, disk crash, or processor overload, the device is notified of that. As a result, the device always knows whether the submitted data is safe to delete or should be resent. Both structured and unstructured data can be collected. It can be of primitive types, such as plain numbers or text, or compound, key-value maps, arrays, or nested objects.

\subsubsection{Data Analytics}

The platform features data collection adapters that allow sending data to various databases or data analytics systems. Owing to its highly modular architecture, a new integration requires minimum effort to modify an existing adapter.

Raw, unstructured data can also be transformed into well-structured time series, convenient for analytics, pattern analysis, visualization, charting, etc. Similarly to raw data, time series can be pushed into a processing or analytics system of our choice.

\subsubsection{Data Visualization}

The data visualization component comprises a rich set of widgets, such as gauges, charts, maps, tables, etc. We can use these widgets to visualize different types of data, whether telemetry, statistics, geo-location, metadata, or other-both historical and current. All widgets are configurable and allow you to change their data sources as well as visual representation.

Besides data visualization, widgets allow us to interact with devices by sending commands, changing configuration and metadata, etc.

\subsubsection{Configuration Management}

Configuration management is essential for controlling the device behavior, managing data processing parameters, edge analytics, feature flagging, and other functions. The platform allows us to implement all of this functionality by providing the configuration management feature that works with arbitrary data structures. Thus, we can apply the configuration data that is as simple as a set of key-values or as complex as nested objects. Since IOT devices might not be constantly connected, the platform tracks already applied configuration data as well as pending delivery.

\subsubsection{Command Execution}

Command execution is the platform feature that allows you to deliver messages with the arbitrary payload to connected devices, execute commands, and receive near-real time responses. For example, we can remotely check current temperature on a home thermostat, point a security camera to a specific area, open a vehicle trunk, and so on.

\section{Application of IOT in Automotive Transportation}

\subsection{Intelligent Fleet Management}

For conventional way of operating the fleet management industry, its productivity and its efficiency has been disrupted with the application of fleet management technology. While more devices, people and data get connected, the opportunities in fleet management are expanding exponentially. The fleet management technology enhances transparency and enables real-time visibility of fleet movement, fuel management, vehicle maintenance, diagnostics, driver management and real-time monitoring. fleet telematics is adopted to maximize safety and efficiency, minimize environmental impact, and keep the cargo secure and in optimal condition. An efficient logistic 
operation which emphasizes loss prevention, and risk management, monitors the supply and demand nerve reacts with agility to market supply and demand requirements.

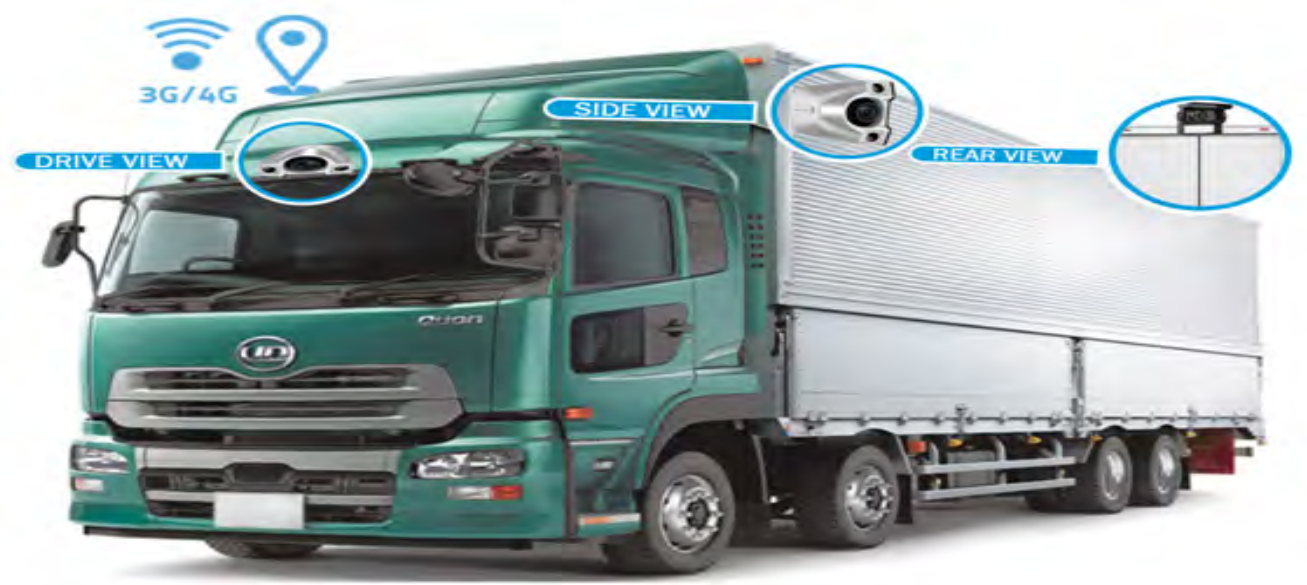

Figure 1. A typical telematic truck

\subsection{Insurance of Operational Optimization, Service Competence of Real Time Tracking Accuracy}

Real-time monitoring with superior resolution, a host of very powerful image processing algorithms and a comprehensive real-time integrated freight data analysis, cloud-based evidence management and onboard storage all packaged in a graphical, user-friendly interface, ensure that the platform conducts complicated monitoring and recording with dependability and reliability.

\subsection{Real- time Video Surveillance on Freight Logistics}

The supply chain is dynamic, growing in size and complexity and is vulnerable to a host of threats and hazards such as natural disasters, accidents, theft and malicious attacks. Linking people, processes and technology together better creates "defensive layers" that makes stealing freight or data along the supply chain a more difficult endeavour. The real time video surveillance cameras combined with proven Video Management System (VMS) provide an end-to-end solution for security, surveillance, evidence and asset management. All information is available through an easy-to-use dashboard interface that works with tablets, smartphones, and computers.

\subsection{Risk Reduction, Operational Costs Decrease and Fleet Safety Improvement}

The vehicle tracking system provides data regarding location, speed, time and direction, and so on. The VTS system can evaluate much more complex information such as, driver behavior analytics with accelerometer-based Three axis Gyro Sensor, monitor harsh braking, rash turning, and harsh acceleration. The VTS system is also proficient in providing data and analytics on fuel efficiency and theft control for liability reduction and insurance to reduce risk, lower operational costs and improve fleet safety. The whole telematics system is the answer to several critical problems like route planning, demand forecasting and predicting delivery time.

\subsection{Advance Driver Assistance Solution (ADAS )}

Advance Driver Assistance Solution (ADAS) is the key to reduction in frequency of road accidents leading to improved vehicle and road safety measures.

The implementation of ADAS technology will result in uncovering the previously undetected risky driver behavioral details. ADAS works with cameras integrated with real-time video data and emergency alarms. ADAS technology significantly helps to monitor and encourage better driver behavior to avoid potential road hazards leading to safer roads and better fleet operational efficiency. 


\section{Conclusion}

The Internet of Things (IOT) helps in smooth connectivity of all vehicles, which not only helps to gain better insight into the driver's behavior but also assists in monitoring the health of the fleet from any device. Rising demand for fleet safety and data management coupled with the growing need to reduce the total cost of ownership (TCO) and achieve fuel efficiency are anticipated to drive the adoption of IOT technology in automotive transportation.

\section{References}

[1]. F. Libo, Y. Honglan, and Z. Xinping, "The design and realization of farmland information monitoring system based on LabVIEW," Journal of Anhui Agricultural Sciences, vol. 39, no. 9, pp. 5563-5565, 2011.

[2]. M. F. Othman and K. Shazali, "Wireless sensor network applications: a study in environment monitoring system,” Procedia Engineering, vol. 41, no. 1, pp. 1204-1210, 2012.

[3]. J. Sun and H. Liu, "Study on fruit cold-chain logistics forewarning model based on BP neural network," Logistics Technology, vol. 32, no. 5, pp. 204-207, 2013.

[4]. J. Wang, H. Wang, J. He et al., "Wireless sensor network for real-time perishable food supply chain management," Computers and Electronics in Agriculture, vol. 110, pp. 196-207, 2015. 